\title{
Ductal carcinoma in situ of the breast - an overview of the randomized clinical trials
}

\author{
Ph. Meijnen, E. J. Th. Rutgers \\ Department of Surgery, The Netherlands Cancer Institute - Antoni van Leeuwenhoek Hospital, \\ Amsterdam, The Netherlands.
}

\begin{abstract}
Since 1985, 5411 patients were randomized in four large randomized clinical trials assessing the role of adjuvant radiotherapy and/or tamoxifen in breast conserving surgery for patients with ductal carcinoma in situ (DCIS).

First the National Surgical Adjuvant Breast and Bowel Project (NSABP) B-17 trial proved relevance of postoperative radiotherapy in breast conserving surgery in DCIS patients: an approximately $50 \%$ reduction of ipsilateral tumour recurrences. This is confirmed by longer time of follow-up and validated by data from the European Organization for Research and Treatment of Cancer (EORTC) 10853 and UK Coordinating Committee on Cancer Research (UKCCCR) DCIS trials. A subset of patients who do not benefit of adjuvant radiotherapy cannot be identified. Completeness of excision proved to be the most important treatment variable to reduce local recurrence.

Both the NSABP B-24 and UKCCCR DCIS trials investigated the effect of tamoxifen resulting in contradictory findings. In addition a possible benefit seems to be restricted to oestrogen receptor-positive DCIS lesions nourishing the existent reserve in prescribing tamoxifen for all patients with DCIS.

The failure to materialize a difference in survival by the effect of either adjuvant radiotherapy or tamoxifen endorses the favourable outcome of DCIS. Currently, four other randomized clinical trials investigate the role of radiotherapy, tamoxifen, anastrazole, or no further treatment for a selected group of patients.
\end{abstract}

Keywords: Clinical trials; Randomized; Ductal carcinoma in situ; Surgery; Breast conserving; Radiotherapy adjuvant; Tamoxifen

\section{Introduction}

Although many people believe the National Surgical Adjuvant Breast and Bowel Project (NSABP) B-17 trial (1985-1990) was the first randomized clinical trial to study the effect of postoperative radiotherapy in patients with breast conserving surgery in ductal carcinoma in situ (DCIS), the NSABP B-06 trial (1976-1984) comparing total mastectomy and

Correspondence to: Emiel J. Th. Rutgers, MD, PhD, Department of Surgery, The Netherlands Cancer Institute - Antoni van Leeuwenhoek Hospital, Plesmanlaan 121, 1066 CX, Amsterdam, The Netherlands. E-mail: e.rutgers@nki.nl; Tel: +31(0)20 512 2551; Fax: +31(0)20 5122554

Publication date 17/12/04

BCO/331/2004/FO segmental mastectomy (lumpectomy) with or without radiotherapy inadvertently included $78 \mathrm{DCIS}$ lesions [1]. Seventy-six of them were followed for 83 months following treatment by lumpectomy only ( $n=21)$ lumpectomy and radiotherapy $(n=27)$, or mastectomy $(n=28)$. Nine of $21(43 \%)$ exhibited recurrences after lumpectomy, two of $27(7 \%)$ after lumpectomy and radiotherapy, and none after mastectomy [2]. The B-17 trial was designed with the same purpose as the B-06 in a period that DCIS had been treated in many different ways, ranging from local excision with or without adjuvant radiotherapy to unilateral or bilateral mastectomy; both studies suggest a beneficial effect of radiation in local control $[3,4]$. In contrast to (and as a result of) the trials 
comparing outcomes after mastectomy with those after breast conserving surgery in invasive breast cancer, it is unlikely that a similar trial for DCIS will ever be designed. Local recurrence rates of $22.5 \%$, $8.9 \%$, and $1.4 \%$ after conservative surgery alone, conservative surgery with adjuvant radiotherapy and mastectomy, respectively were reported in a metaanalysis of 4174 patients [5]. Lack of difference in survival between these treatment modalities led to the intricacy of patient tailored management in DCIS. Considering invasive recurrence as the most potent violator of local failure and keeping in mind the possibility of a salvage mastectomy, breast conserving surgery should - in general - be the standard treatment for all DCIS lesions. Unfortunately, medicine is not so straightforward since DCIS lesions are very heterogenous in their presence and behaviour. Multicentric and large lesions are not uncommon and bear a higher risk of harbouring a (micro) invasive component. So far, four large randomized clinical trials have been designed and carried out to evaluate the role of adjuvant radiotherapy and/or hormonal treatment in breast conserving therapy of DCIS patients: the NSABP B-17 and B-24 trials, the European Organization for Research and Treatment of Cancer (EORTC) 10853 trial, and the recent UK Coordinating Committee on Cancer Research (UKCCCR) DCIS trial (Table 1).

\section{NSABP B-17 trial}

The NSABP B-17 trial was the first large trial to investigate the effect of postoperative radiotherapy in patients diagnosed with this noninvasive lesion. Primary endpoints were ipsilateral, contralateral, regional, or distant recurrences. From 1985-1990, a group of 818 women with DCIS were randomly assigned to undergo lumpectomy or lumpectomy followed by radiotherapy (50 Gy). Eighty-one per cent of all lesions were detected by mammography. Hence, the term lumpectomy is obsolete for most of the procedures where no palpable lump could be detected. Margins of the resected specimen were histologically tumour-free, defined as tumour-filled ducts not touching an inked surface. Axillary dissection was obligatory at the onset of the study, but subsequently became optional on the basis of evidence, indicating that it was not necessary in the treatment of DCIS [6]. Three hundred (38\%) patients underwent an axillary dissection ultimately. Results after 3.6 years of median follow-up were reported in 1993 leading to the recommendation that radiotherapy after lumpectomy is more appropriate than lumpectomy alone for women with localized DCIS: $7 \%$ vs. $16.4 \%$ risk of ipsilateral tumour recurrence, respectively $(P<0.001)[4]$. These findings were affirmed by longer time of follow-up: $12.1 \%$ vs. $26.8 \%$ at 8 years [7] and $15.7 \%$ vs. $31.7 \%$ at 12 years, respectively [8]. Invasive ipsilateral recurrence rate was reduced by $47 \%(P=0.01)$, and noninvasive by $15 \%(P=0.48)$. In total, 413 patients were assigned to radiotherapy resulting in 29 invasive, and 32 noninvasive ipsilateral local recurrences compared to 405 patients treated with excision alone resulting in 66 invasive, and 57 noninvasive ipsilateral local recurrences. The 12-year local recurrence free survival rate was $84.3 \%$ and $68.3 \%$ for the radiotherapy and excision alone group, respectively. Overall survival was $86 \%$ for the patients who underwent radiotherapy and $87 \%$ for the patients treated with excision alone.

First analysis of possible pathological discriminants predictive of ipsilateral recurrence resulted in the association of positive margins and comedonecrosis with higher risk for ipsilateral recurrence [9]. Nevertheless, these features failed in selecting a subgroup of patients who benefit most of adjuvant radiotherapy. Following this first large DCIS trial margin width has become subject of many studies [10-12] but its role in selecting patients has not yet been confirmed by a prospective study. An abstract on the 26th Annual San Antonio Breast Cancer Symposium in 2003 reported the findings of an early stopped prospective study investigating the role of local excision alone with margins of $\geqslant 1 \mathrm{~cm}$ for small, Grade 1 or 2 DCIS. The accrual goal of 200 patients could not be achieved as the study was closed to further accrual at 157 patients because of the high number of ipsilateral local recurrences (5 year rate: $12.5 \%$ ) suggesting benefit of radiotherapy for all patients treated with conservation [13]. The B-17 trial was criticized on the histological data reflecting the apparent assumption that all DCIS is histologically the same [14]. However, the heterogeneity of this lesion became more evident $[15,16]$. Secondly, partial tissue sampling in this trial may have harboured a risk of missing invasion. Further, dichotomous stratification of grade and size hampered comparison with other studies, and made it likely that many cases were larger and invasive. In addition, the definition of histologically free margins was doubtful leading to residual disease in many cases which made it more difficult to assess the benefit of radiotherapy [14]. An update of the pathological findings reported in 1995 was published in 1999 and represented the results of 623 patients [17]. Unlike the first analysis of the B-17 study, uncertain/involved margins became a borderline risk factor for ipsilateral recurrence at 8 years, although, its early importance resembles the need for clear margins. The most favourable subgroup, comprised of patients with absent or slight comedonecrosis and negative 


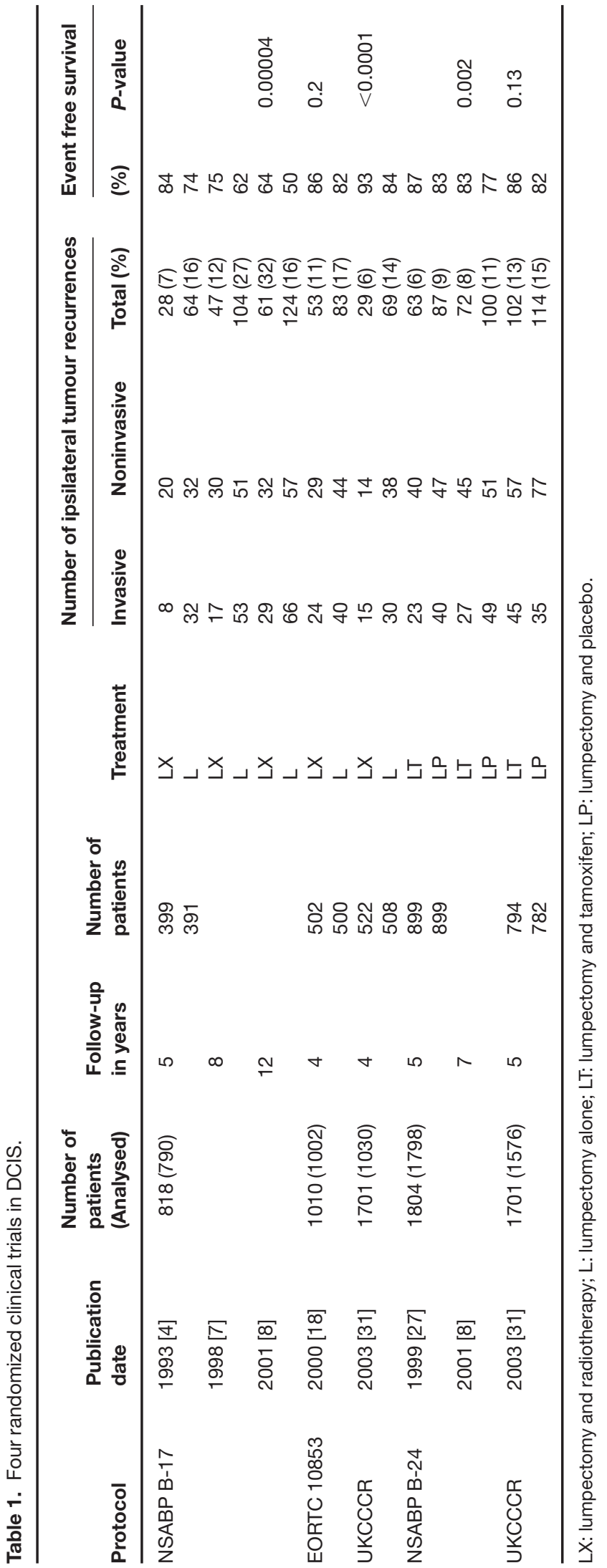


margins, still demonstrated a $7 \%$ reduction in recurrence at 8 years leading to the conclusion that the use of adjuvant radiotherapy is appropriate for all patients with DCIS.

\section{EORTC 10853 trial}

Identical in study design and results is the EORTC 10853 trial which randomized 1010 patients by 46 institutes from 13 different countries between 1986 and 1996. Seventy-one per cent were detected by mammography only. At a median follow-up of 4.25 years, $11 \%$ of the patients treated with radiotherapy developed an ipsilateral recurrence compared to $17 \%$ of the patients without further treatment [18]. Five hundred and seven patients received radiotherapy resulting in the development of 24 invasive, and 29 noninvasive ipsilateral recurrences. Five hundred and three patients were assigned to no further treatment of which 40 patients developed an invasive ipsilateral recurrence, and 44 patients a noninvasive ipsilateral recurrence. The 4-year local recurrence free survival rate was $91 \%$ vs. $84 \%$ in the radiotherapy and excision alone group, respectively. These findings confirm the favourable effect of radiotherapy in the conservative treatment of DCIS as reported by the NSABP B-17 $[4,7,8]$. In view of the comparable follow-up of the B-17 data published in 1993, the effect on the reduction of invasive ipsilateral recurrence was larger in the American B-17 trial (2.9\% vs. $4 \%)$ than in the EORTC study. The findings in nonrandomized studies that about half of all local recurrences after conservative treatment with radiotherapy are invasive corresponds with the EORTC findings [11,19-21]. The observation of a significant higher rate of contralateral breast cancers diagnosed in the radiotherapy group of the 10853 trial could not be clearly explained. Epidemiological data indicate that the median induction period after radiotherapy for Hodgkin's disease is around 15 years (range 4-20) [22] suggesting another cause or, more likely, a result of chance. The 8 years update of the B-17 trial reported also a higher, but not significant, rate of contralateral breast cancer occurrence reported as first event while the overall number of contralateral breast cancers was equally distributed. The risk of eventually dying from breast cancer when DCIS is treated with conservative surgery is still minimal: four patients died of ipsilateral recurrence in the radiotherapy group, and seven patients in the no further treatment group. The 4-year overall survival rate was $99 \%$. Longer follow-up is needed to investigate whether the beneficial effect of radiotherapy on local control will improve survival rates.

In search of risk factors predictive for invasive recurrence a central review of 863 (85\%) patients was undertaken: in 10\% DCIS could not be confirmed. Five per cent of the patients were classified as benign proliferative lesions, $1.5 \%$ as suspicious of invasion, and $3 \%$ as invasive [23]. In the B-17 trial $2 \%$ invasive lesions were found [9]. In the EORTC study lesions classified as atypical ductal hyperplasia did not come across compared to $7 \%$ in the $\mathrm{B}-17$ trial. This likely reflects the existing problems with the classification of DCIS [24]. Further, as result of the finding that no recurrences were observed in 59 patients with well-differentiated DCIS with a clinging architecture, the authors seemed it justified to classify this group of lesions as 'columnar alteration with prominent apical snouts and secretion', or 'atypical ductal cells with apocrine snouts', and recommended close follow-up and excision only for these favourable lesions. Poorly defined margin status in the study design did not require the assessment of margin width. For this reason the opportunity to select patients eligible for excision alone was missed. Factors significantly associated with recurrence were age ( $\leqslant 40$ years), cribriform or solid/ comedo architecture, symptomatic detection, and close/involved or unknown margins. In addition, poorly differentiated DCIS was correlated with noninvasive local recurrence. Further, relapses of poorly differentiated DCIS was more frequently associated with distant metastasis and death after invasive local recurrence.

\section{NSABP B-24 trial}

The NSABP B-24 protocol was written after the observation that many women were ineligible for the B-17 trial because they had been treated by mastectomy in case of extensive calcifications, or because margins remained positive after multiple excisions. In addition, the favourable effect of hormonal treatment by tamoxifen for patients with invasive breast cancer was already reported $[25,26]$ but not yet investigated for DCIS. Between 1991 and 1994, 1804 patients, including those whose margins were involved with tumour were randomized for lumpectomy, radiotherapy (50 Gy), and placebo, or tamoxifen ( $20 \mathrm{mg}$ daily for 5 years) instead of placebo. Sixteen per cent of the patients had positive margins and in $10 \%$ margins were uncertain. Thirty-one per cent $(n=564)$ of the patients showed a lack of compliance because of side effects ( $n=146$ in tamoxifen group, $n=98$ in placebo group), personal reasons ( $n=124$ in tamoxifen group, $n=146$ in placebo group), or unspecified reasons ( $n=25$ in both groups). Seventy per cent of all breast cancer events were ipsilateral. The likelihood of ipsilateral tumour recurrence at 5 years of follow-up was $6 \%$ after administration of tamoxifen and $9 \%$ after placebo $(P=0.04)$ [27]. The latter was 
almost identical with the 5 years result of the B-17 trial indicative for the relationship between these two trials [9]. Seven years of follow-up demonstrated an $8 \%$ (3\% invasive, $5 \%$ noninvasive) ipsilateral recurrence rate after tamoxifen compared to $11 \%(5 \%$ invasive, $6 \%$ noninvasive) with placebo $(P=0.02)$ [8]. Noninvasive local recurrences seem unaffected by the administration of tamoxifen $(P=0.48)$. In addition, the benefit seems to be restricted to oestrogen receptor (ER)-positive DCIS lesions with a relative risk of $0.41(P<0.001)$ in the ER-positive group compared to $0.8(P=0.51)$ in the ER-negative group. ER status was determined for 628 patients of which $482(77 \%)$ were positive [28]. Contralateral breast cancer as first event was reduced with $53 \%$ $(P=0.01)$. In total, 39\% fewer breast cancer events were observed in the tamoxifen group. Young age ( $\leqslant 49$ years) was associated with local recurrence, as were positive margins, symptomatic detected lesions, and comedonecrosis. Systemic treatment with tamoxifen showed no survival benefit so far (95\% in both groups after 7 years).

The NSABP P-1 trial demonstrated that tamoxifen reduced the incidence of invasive, and noninvasive cancer in high-risk populations [29]. Patients with a history of atypical ductal hyperplasia or lobular carcinoma in situ had about a 50\% reduction in the risk of invasive breast cancer overall. Acceptance of the profit of this intervention for these high-risk patients cannot be taken without the consideration of administration of tamoxifen for DCIS patients as well; even if they had been treated with radiotherapy. Since patients with DCIS have twice a higher risk of invasive breast cancer than those with lobular carcinoma in situ, and an even three times higher risk than patients with atypical ductal hyperplasia lesions [27].

The occurrence of adverse events included nine cases of deep vein thrombosis and two cases of nonfatal pulmonary embolism in the tamoxifen group vs. two cases and one case, respectively, in the placebo group. These numbers were expanded with a report of six strokes (five tamoxifen, one placebo) [30]. The P-1 study showed a nonsignificant increase in the number of strokes for patients receiving tamoxifen. Although endometrial cancer was increased in the tamoxifen arm compared to the placebo, total number of events were minimal (1.53 vs. 0.45 per 1000 patients per year, respectively) in the B-24 study. The toxicity profile of tamoxifen indicates selection of patients who have the least risk of serious side effects.

\section{UKCCCR DCIS trial}

A second randomized trial to assess the role of tamoxifen in combination with adjuvant radiotherapy or not, for the treatment of patients with DCIS was set up by the UKCCCR (now the National Cancer Research Institute [NCRI]) DCIS working party in collaboration with the Australian-New Zealand Breast Cancer Trials Group, and consisted of a $2 \times 2$ factorial design. Again, the primary endpoint was invasive ipsilateral recurrence. Between 1990 and 1998, 1701 patients were randomized to both treatments in combination or singly, or to none, or to either one with an elective decision to give or to withhold the other. Complete surgical excision of the lesion was confirmed by specimen radiography and histology. There was no evidence of an interaction for any of the endpoints. After a median follow-up of 4.4 years, ipsilateral tumour recurrence was reduced from $15 \%$ to $13 \%(P=0.42)$ by the prescription of tamoxifen at a dose of $20 \mathrm{mg}$ daily for 5 years. Eleven per cent of the patients in the tamoxifen arm did not take the treatment for the complete 5 years. The comparison of radiotherapy and no radiotherapy revealed an ipsilateral tumour recurrence of $6 \%$ and $14 \%(P<0.0001)$ in favour of adjuvant radiotherapy [31]. In contrast to the B-24, the UKCCCR DCIS trial did not demonstrate a favourable effect of tamoxifen contributing the reserve in prescribing this hormonal agent for DCIS patients. This could be explained by a difference in distribution of age between the two trials demonstrating at least a beneficial effect of hormonal administration in patients $<50$ years of age in both studies. Authors' reply on complaints about the lack of histological information $[32,33]$ added a possible explanation for the failure in validating the B-24 results based on a difference in the histological characteristics of lesions from patients entered in to these two trials. The higher frequency of high-grade lesions included in the UKCCCR trial is likely a result from differences in clinical assessment and management of screen-detected lesions. High-grade DCIS has a lower frequency of ER positivity than do low- or intermediate-grade DCIS, which would explain the differences in proportions of hormone-receptor status in these two trials and consequently the differences in tamoxifen effect. In view of the possible negative effects of tamoxifen: two endometrial, four ovarian, and two unspecified gynaecological tumours were reported; only one did not receive tamoxifen as adjuvant treatment. This difference was not significant. No other adverse events were reported. A central histopathological review is undertaken and the results are eagerly awaited.

\section{Recently closed and ongoing trials}

\section{ECOG E-5194}

The American Eastern Cooperative Oncology Group (ECOG) E-5194 trial investigates local excision alone 
for patients with DCIS lesions that were $\leqslant 2.5 \mathrm{~cm}$ and low or intermediated grade, or $\leqslant 1 \mathrm{~cm}$ and high grade. Margins width of at least $3 \mathrm{~mm}$ was necessary for all patients. Approximately 1000 patients were included to close this trial. Five- and ten-year local recurrence rates will hopefully provide more information on the efficacy of lumpectomy alone for low-risk DCIS leading to criteria for selecting patients who do not need adjuvant radiotherapy [34].

\section{RTOG 9804}

Still ongoing is the Radiation Therapy Oncology Group (RTOG) 9804 study. Patients are assigned either to adjuvant radiation therapy or not with the option of tamoxifen. Lesions must be $\leqslant 2.5 \mathrm{~cm}$ in diameter, low or intermediate grade, and have a minimum margin width of $3 \mathrm{~mm}$. In total 1790 are required [34].

\section{NSABP B-35 trial}

The development of newer hormonal drugs has led to the start of the NSABP-B35 trial in January 2003. ER-positive postmenopausal patients are assigned to adjuvant anastrozole (aromatase inhibitor) or tamoxifen after local therapy. Unlike tamoxifen, anastrazole actually prevents the production of oestrogen in postmenopausal women with fewer side effects. Anastrazole has already proven its efficacy in postmenopausal patients with hormone-sensitive invasive breast cancer [35]. In total, 3500 postmenopausal patients with ER-positive DCIS lesions will be randomized to adjuvant anastrazole or tamoxifen. Primary endpoints are local recurrence and the development of contralateral breast cancer.

\section{IBIS-II trial}

A similar background as the previous trial has the International Breast Cancer Intervention Study (IBIS-II) which started in September 2003. The IBISII trial aims to identify the role of the relatively new drug anastrazole in 10000 women at a higher risk of developing breast cancer including 4000 patients with DCIS. Women at high risk are randomized to anastrazole vs. nil, while patients with ER-positive DCIS are randomized between anastrazole and tamoxifen after complete excision and radiotherapy.

\section{Conclusion}

Important take-home messages are as follows:

1. Adjuvant radiotherapy reduces significantly the incidence of ipsilateral recurrences after breast conserving surgery in patients with completely excised DCIS.

2. A subset of low-risk patients cannot be selected to whom radiotherapy is of no benefit.

3. A complete excision is the most important treatment variable to reduce recurrences.

4. The administration of tamoxifen should be reserved for patients at higher risk for local relapse and who would like to preserve the breast.

5. There is not a difference in survival between the adjuvant treatment modalities indicative for the relative favourable prognosis of most DCIS lesions.

\section{References}

1. Fisher ER, Sass R, Fisher B, Wickerham L, Paik SM. Pathologic findings from the National Surgical Adjuvant Breast Project (protocol 6). I. Intraductal carcinoma (DCIS). Cancer 1986; 57: 197-208.

2. Fisher ER, Leeming R, Anderson S, Redmond C, Fisher B. Conservative management of intraductal carcinoma (DCIS) of the breast. Collaborating NSABP investigators. J Surg Oncol 1991; 47: 139-147.

3. Fisher B, Redmond C, Poisson R, Margolese R, Wolmark N, Wickerham L, et al. Eight-year results of a randomized clinical trial comparing total mastectomy and lumpectomy with or without irradiation in the treatment of breast cancer. New Engl J Med 1989; 320: 822-828.

4. Fisher B, Costantino J, Redmond C, Fisher E, Margolese R, Dimitrov N, et al. Lumpectomy compared with lumpectomy and radiation therapy for the treatment of intraductal breast cancer. New Engl J Med 1993; 328: 1581-1586.

5. Boyages J, Delaney G, Taylor R. Predictors of local recurrence after treatment of ductal carcinoma in situ: a meta-analysis. Cancer 1999; 85: 616-628.

6. Silverstein MJ, Rosser RJ, Gierson ED, Waisman JR, Gamagami P, Hoffman RS et al. Axillary lymph node dissection for intraductal breast carcinoma - is it indicated? Cancer 1987; 59: 1819-1824.

7. Fisher B, Dignam J, Wolmark N, Mamounas E, Costantino J, Poller W, et al. Lumpectomy and radiation therapy for the treatment of intraductal breast cancer: findings from National Surgical Adjuvant Breast and Bowel Project B-17. J Clin Oncol 1998; 16: 441-452.

8. Fisher B, Land S, Mamounas E, Dignam J, Fisher ER, Wolmark N. Prevention of invasive breast cancer in women with ductal carcinoma in situ: an update of the national surgical adjuvant breast and bowel project experience. Semin Oncol 2001; 28: 400-418.

9. Fisher ER, Costantino J, Fisher B, Palekar AS, Redmond C, Mamounas E. Pathologic findings from the National Surgical Adjuvant Breast Project (NSABP) Protocol B-17. Intraductal carcinoma (ductal carcinoma in situ). The National Surgical Adjuvant Breast and Bowel Project Collaborating Investigators. Cancer 1995; 75: 1310-1319.

10. Silverstein MJ, Lagios MD, Groshen S, Waisman JR, Lewinsky BS, Martino S, et al. The influence of margin width on local control of ductal carcinoma in situ of the breast. New Engl J Med 1999; 340: 1455-1461. 
11. Solin LJ, Fourquet A, Vicini FA, Haffty B, Taylor M, McCormick B, et al. Mammographically detected ductal carcinoma in situ of the breast treated with breastconserving surgery and definitive breast irradiation: longterm outcome and prognostic significance of patient age and margin status. Int J Radiat Oncol Biol Phys 2001; 50: 991-1002.

12. Kerlikowske K, Molinaro A, Cha I, Ljung BM, Ernster VL, Stewart $\mathrm{K}$, et al. Characteristics associated with recurrence among women with ductal carcinoma in situ treated by lumpectomy. J Natl Cancer Inst 2003; 95: 1692-1702.

13. Wong JS, Gadd MA, Gelman R, Kaelin CM, Lester S, Schnitt SJ, et al. Wide excision alone for ductal carcinoma in situ (DCIS) of the breast. Breast Cancer Res Treat 2003; 82[Suppl 1]: s10.

14. Page DL, Lagios MD. Pathologic analysis of the National Surgical Adjuvant Breast Project (NSABP) B-17 Trial. Unanswered questions remaining unanswered considering current concepts of ductal carcinoma in situ. Cancer 1995; 75: 1219-1222.

15. Lennington WJ, Jensen RA, Dalton LW, Page DL. Ductal carcinoma in situ of the breast. Heterogeneity of individual lesions. Cancer 1994; 73: 118-124.

16. Patchefsky AS, Schwartz GF, Finkelstein SD, Prestipino A, Sohn SE, Singer JS, et al. Heterogeneity of intraductal carcinoma of the breast. Cancer 1989; 63: 731-741.

17. Fisher ER, Dignam J, Tan-Chiu E, Costantino J, Fisher B, Paik $S$, et al. Pathologic findings from the National Surgical Adjuvant Breast Project (NSABP) eight-year update of Protocol B-17: intraductal carcinoma. Cancer 1999; 86, 429-438.

18. Julien JP, Bijker N, Fentiman IS, Peterse JL, Delledonne V, Rouanet $\mathrm{P}$, et al. Radiotherapy in breast-conserving treatment for ductal carcinoma in situ: first results of the EORTC randomised phase III trial 10853. EORTC Breast Cancer Cooperative Group and EORTC Radiotherapy Group. Lancet 2000; 355: 528-533.

19. Amichetti M, Caffo O, Richetti A, Zini G, Rigon A, Antonello $\mathrm{M}$, et al. Ten-year results of treatment of ductal carcinoma in situ (DCIS) of the breast with conservative surgery and radiotherapy. Eur J Cancer 1997; 33: 1559-1565.

20. Cutuli B, Cohen-Solal-Le Nir C, De Lafontan B, Mignotte $\mathrm{H}$, Fichet V, Fay $\mathrm{R}$, et al. Ductal carcinoma in situ of the breast results of conservative and radical treatments in 716 patients. Eur J Cancer 2001; 37: 2365-2372.

21. Silverstein MJ, Barth A, Poller DN, Gierson ED, Colburn WJ, Waisman JR, et al. Ten-year results comparing mastectomy to excision and radiation therapy for ductal carcinoma in situ of the breast. Eur $J$ Cancer 1995; 31A: 1425-1427.

22. Ralleigh G, Given-Wilson R, On Behalf Of The Royal College Of Radiologists Breast Group Working Party On Hodgkin's Disease And Secondary Breast Cancer. Breast cancer risk and possible screening strategies for young women following supradiaphragmatic irradiation for Hodgkin's disease. Clin Radiol 2004; 59: 647-650.
23. Bijker N, Peterse JL, Duchateau L, Julien JP, Fentiman IS, Duval C, et al. Risk factors for recurrence and metastasis after breast-conserving therapy for ductal carcinoma-in-situ: analysis of European Organization for Research and Treatment of Cancer Trial 10853. $J$ Clin Oncol 2001; 19: 2263-2271.

24. van de Vijver MJ, Peterse $\mathrm{H}$. The diagnosis and management of pre-invasive breast disease: pathological diagnosis - problems with existing classifications. Breast Cancer Res 2003; 5: 269.

25. Fisher B, Costantino J, Redmond C, Poisson R, Bowman D, Couture J, et al. A randomized clinical trial evaluating tamoxifen in the treatment of patients with node-negative breast cancer who have estrogen-receptorpositive tumors. New Engl J Med 1989; 320: 479-484.

26. Fisher B, Redmond C. New perspective on cancer of the contralateral breast: a marker for assessing tamoxifen as a preventive agent. J Natl Cancer Inst 1991; 83: 1278-1280.

27. Fisher B, Dignam J, Wolmark N, Wickerham DL, Fisher ER, Mamounas E, et al. Tamoxifen in treatment of intraductal breast cancer: National Surgical Adjuvant Breast and Bowel Project B-24 randomised controlled trial. Lancet 1999; 353: 1993-2000.

28. Allred DC, Bryant J, Land S, Paik S, Fisher E, Julian T, et al. Estrogen receptor expression as a predictive marker of the effectiveness of tamoxifen in the treatment of DCIS: Findings from NSABP Protocol B-24. Breast Cancer Res Treat 2003; 76: s36.

29. Fisher B, Costantino JP, Wickerham DL, Redmond CK, Kavanah M, Cronin WM, et al. Tamoxifen for prevention of breast cancer: report of the National Surgical Adjuvant Breast and Bowel Project P-1 Study. J Natl Cancer Inst 1998; 90: 1371-1388.

30. Dignam JJ, Fisher B. Occurrence of stroke with tamoxifen in NSABP B-24. Lancet 2000; 355: 848-849.

31. Houghton J, George WD, Cuzick J, Duggan C, Fentiman IS, Spittle M. Radiotherapy and tamoxifen in women with completely excised ductal carcinoma in situ of the breast in the UK, Australia, and New Zealand: randomised controlled trial. Lancet 2003; 362: 95-102.

32. Cunnick GH, Mokbel K. Radiotherapy and tamoxifen in women with completely excised ductal carcinoma in situ. Lancet 2003; 362: 1154-1156.

33. Zammit C, Yelland A, Deutsch G. Radiotherapy and tamoxifen in women with completely excised ductal carcinoma in situ. Lancet 2003; 362: 1154-1156.

34. Leonard GD, Swain SM. Ductal carcinoma in situ, complexities and challenges. J Natl Cancer Inst 2004; 96: 906-920.

35. Baum M, Budzar AU, Cuzick J, Forbes J, Houghton JH, Klijn JG, et al. Anastrozole alone or in combination with tamoxifen versus tamoxifen alone for adjuvant treatment of postmenopausal women with early breast cancer: first results of the ATAC randomised trial. Lancet 2002; 359: 2131-2139. 